\title{
Philosophiques
}

\section{Nature et fonction de la mémoire dans À la recherche du temps perdu}

\section{Jacques J. Zéphir}

Volume 17, numéro 2, automne 1990

URI : https://id.erudit.org/iderudit/027123ar

DOI : https://doi.org/10.7202/027123ar

Aller au sommaire du numéro

\section{Éditeur(s)}

Société de philosophie du Québec

\section{ISSN}

0316-2923 (imprimé)

1492-1391 (numérique)

Découvrir la revue

Citer cet article

Zéphir, J. J. (1990). Nature et fonction de la mémoire dans À la recherche du temps perdu. Philosophiques, 17(2), 147-168. https://doi.org/10.7202/027123ar
Résumé de l'article

Dans À la recherche du temps perdu, Proust est, en réalité, à la recherche de son identité, de son moi profond et véritable. Pour ce faire, il s'isole du présent dans le but de se retrouver dans le passé. Cependant, la «résurrection du passé ", qui doit lui apporter le salut éperdument recherché, n'est pas le produit de la mémoire volontaire. Cette forme de mémoire, fonction de l'évocation objective et " quasi-dépersonnalisée », n'a pas, au dire de Proust, le don de nous représenter le passé dans sa plénitude totale. Dès lors, elle ne saurait le conduire au moi profond, dont elle ne pourrait lui donner qu'une image factice et tronquée. En revanche, la mémoire authentique, selon Proust, est la mémoire involontaire, qui est liée à l'actualité de notre vie par la plus mystérieuse des intimités. Et même, à l'en croire, cette mémoire aurait une fonction méta- physique précise, qui serait de nous révéler notre moi profond, de faire émerger, à la surface de la monotonie de notre présent, notre « essence ». C'est que, pour Proust, le souvenir involontaire n'est pas, en réalité, quelque chose qu'on a, mais bien quelque chose qu'on est. Son rôle n'est pas seulement de fixer des événements écoulés, mais de nous exprimer nous-même, d'affirmer, par delà la multiplicité de nos moi changeants et superficiels, l'identité et la permanence d'un moi authentique et profond.
Tous droits réservés (C) Société de philosophie du Québec, 1990
Ce document est protégé par la loi sur le droit d'auteur. L’utilisation des services d'Érudit (y compris la reproduction) est assujettie à sa politique d'utilisation que vous pouvez consulter en ligne.

https://apropos.erudit.org/fr/usagers/politique-dutilisation/ 


\title{
NATURE ET FONCTION DE LA MÉMOIRE DANS À LA RECHERCHE DU TEMPS PERDU
}

\author{
par Jacques J. Zéphir
}

RÉSUMÉ. Dans $\dot{A}$ la recherche du temps perdu, Proust est, en réalité, à la recherche de son identité, de son moi profond et véritable. Pour ce faire, il s'isole du présent dans le but de se retrouver dans le passé. Cependant, la * résurrection du passé *, qui doit lui apporter le salut éperdument recherché, n'est pas le produit de la mémoire volontaire. Cette forme de mémoire, fonction de l'évocation objective et "quasi-dépersonnalisée", n'a pas, au dire de Proust, le don de nous représenter le passé dans sa plénitude totale. Dès lors, elle ne saurait le conduire au moi profond, dont elle ne pourrait lui donner qu'une image factice et tronquée. En revanche, la mémoire authentique, selon Proust, est la mémoire involontaire, qui est liée à l'actualité de notre vie par la plus mystérieuse des intimités. Et même, à l'en croire, cette mémoire aurait une fonction métaphysique précise, qui serait de nous révéler notre moi profond, de faire émerger, à la surface de la monotonie de notre présent, notre "essence». C'est que, pour Proust, le souvenir involontaire n'est pas, en réalité, quelque chose qu'on $a$, mais bien quelque chose qu'on est. Son rôle n'est pas seulement de fixer des événements écoulés, mais de nous exprimer nous-même, d'affirmer, par delà la multiplicité de nos moi changeants et superficiels, l'identité et la permanence d'un moi authentique et profond.

ABSTRACT. In Remembrance of Things Past (ì la recherche du temps perdu), Proust is actually searching for his own identity, his innermost, true self. In order to do this, he isolates himself from the present, with the aim of finding himself in the past. However, the "resurrection of the past ", which ought to bring him the salvation so desperately sought, is not the product of voluntary memory. This form of memory, function of the objective, "quasi-depersonalized * evocation, does not have the capability of presenting the past to us again in its totality. Consequently, it could not lead him to the innermost self, of which it could give him only a factitious and truncated image. 
On the other hand, the authentic memory is the involuntary memory, which is linked to the reality of our life by the most mysterious connections. This memory, according to Proust, would even have a precise metaphysical function, which would be to reveal to us our innermost self, to make our "essence* emerge at the surface of the monotony of our present. This means that the involuntary remembrance is not, in reality, something that one bas, but rather something that one is. Its role is not only to set events in time, but to explain ourself to us, to affirm, over and above the multiplicity of our changing and superficial selves, the identity and permanence of an authentic, innermost self. 
"Il est regrettable que les philosophes ne lisent pas plus souvent des romans. Du moins, c'est ce qu'on serait tenté de dire lorsqu'on s'avise de la minceur du vocabulaire utilisé dans la philosophie morale d'aujourd'hui. Je corrigerai pourtant le regret énoncé ci-dessus : il est dommage que les philosophes ne parlent pas plus abondamment des romans qu'ils lisent.

Les philosophes ont le plus grand besoin de lire des romans s'il est vrai que la forme romanesque est aujourd'hui la plus riche en legomena, en échantillons de ces manières communes de penser qui sont la lumière première de la philosophie pratique."

Vincent Descombes,

Proust, pbilosophe du roman, Éditions de Minuit.

C'est une Conviction Chez Proust, - l'expérience le lui a montré, - que le présent et le réel ne nous livrent que la surface des êtres et des choses et nous fixent nous-mêmes à notre propre superficie. Ce qui compte, c'est «le passé dont les choses gardent l'essence, et l'avenir où elles nous incitent à le goûter de nouveau " ${ }^{1}$. Se plonger dans le passé nous remplit d'une douceur et d'un bonheur infinis, à cause de ce qu'il a d'irréalisable et pourtant de vrai, puisqu'il représente la totalité de notre vie, baignée dans la plus pure réalité spirituelle. Ce caractère réel, bien que spirituel, n'appartient qu'aux choses du passé ; nous ne le retrouvons à l'état pur ni dans le présent, ni dans l'avenir. C'est en se penchant sur son passé que l'esprit retrouve ce que signifie pour lui vivre, s'épanouir, se réaliser.

Le passé seul, "essence intime de nous-mêmes", est donc, selon Proust, capable de nous rendre notre moi permanent et absolu. Enfermés dans le présent, nous sommes soumis à la mort fragmentaire et successive de nos moi. Il faut à notre esprit du recul pour que s'ouvrent à lui et s'éclairent, en certaines du moins de ses évocations du passé, les immatérielles profondeurs, d'abord soustraites à son regard.

Même pour la duchesse de Guermantes, comme pour certaines pages de Bergotte [écrit Proust] son charme ne m'était visible qu'à distance et s'évanouissait quand j'étais près d'elle, car il résidait dans ma mémoire. ${ }^{2}$

Ainsi, ce qu'il y a dans le réel et le présent de richesse et de joie tient surtout à ce qui y survit du passé. Notre 
perception déforme la réalité présente: "nous voyons, nous entendons, nous concevons le monde tout de travers" ${ }^{3}$, et c'est là une perpétuelle cause d'erreur dont les effets s'exercent non seulement sur l'univers visible, sur la vie sociale, mais encore sur la vie intérieure. Si bien qu'au fond, le présent ne se réalise vraiment pour nous qu'une fois devenu le passé. Et la réalité, nous devons la rechercher, non pas dans la perception présente, mais dans la mémoire, car dans celle-ci, la réalité est décantée de toute considération utilitaire et revêt toujours "un charme qui lui vient de la mémoire même et de n'être pas perçu par les sens" 4 . "Les fleurs qu'on me montre aujourd'bui pour la première fois, affirme Proust, ne me semblent pas de vraies fleurs [...]. La réalité ne se forme que dans la mémoire " 5 . Ailleurs, il parle encore de "la terrible puissance recréatrice de la mémoire " ${ }^{6}$. C'est donc à la mémoire, d'après lui, que nous devrions nos impressions les plus vraies, à la mémoire qui est le propre de l'homme :

... cet être sans âge, et être qui a la faculté de redevenir en quelques secondes de beaucoup d'années plus jeune, et qui entouré des parois du temps où il a vécu, y flotte, mais comme dans un bassin dont le niveau changerait constamment et le mettrait à portée tantôt d'une époque, tantôt d'une autre. ${ }^{7}$

Dédaignant donc le moment actuel, Proust s'isole du présent pour se rejoindre, se retrouver dans le passé, qui contient sa vie et l'essence même de son être. Immobile dans sa chambre, jalousement défendu contre le dehors et pareil à un patient aux yeux clos qui laisse venir à la surface de son être des aveux endormis, Proust se redonne son passé. Et, au lieu de se borner à le considérer tout simplement, il renaît à la vie antérieure, il lui fait place, il l'accueille au-dedans de lui dans sa plénitude. ${ }^{8}$ Comme Bergson, il est convaincu que,

3. III (F), 573.

4. I (S), 98.

5. Ibid., p. 184.

6. lbid., p. 368.

7. III (F), 613-14.

8. À ce propos, voici ce que dit Madeleine Remacle : “Chez Proust, l'introspection est toujours en éveil; elle s'exerce avec une vigilance exceptionnelle sur les phénomènes quotidiens et en apparence insignifiants de notre vie sensible. États d'inconscience, états de demi-conscience attirent ce psychologue avide de pénétrer les coucbes les plus profondes du moi. Il fouille en lui-même pour $y$ deceler le plus intime, le plus spécifique ment individuel, pour le faire vivre, pour le comprendre, le définir, et enfin le réduire à quelque grande loi dont chacun puisse trouver en soi l'application. * (Madeleine REMACLE, L'Élément poétique dans *À la recherche du temps perdu * de Marcel Proust, Bruxelles, Palais des Académies, 1954, p. 18. 
derrière les souvenirs qui viennent se poser sur notre occupation présente, il y en a d'autres, des milliers et des milliers d'autres, en bas, au-dessous de la scène illuminée par la conscience. 9

Il sait que dans ses souvenirs sa vie passée est là, conservée jusque dans ses moindres détails, et que ceux-ci attendent qu'il vienne écarter l'obstacle, qu'il soulève la trappe qui les maintenait dans le sous-sol de la conscience pour entrer en mouvement et remonter au champ de la conscience.

POURTANT, la "résurrection envabissante du passé ", qui doit apporter à l'analyste le salut éperdument recherché après des années, n'est pas le produit de n'importe quelle mémoire. Cette faculté humaine a diverses formes, sous lesquelles elle perd toute aptitude à ressusciter effectivement le passé dans sa vérité et sa pureté intégrales. Proust ne le sait que trop bien. Aussi, dans son œuvre, distingue-t-il bien la mémoire volontaire de la mémoire involontaire ${ }^{10}$.

À son sens, la mémoire volontaire "qui est surtout une mémoire de l'intelligence et des yeux ne nous donne du passé que des faces sans vérité "11. Travaillant sur les souvenirs bruts que l'intelligence doit analyser, classer, assimiler, cette forme de mémoire ne rend pas le passé dans son in tégralité et sa pureté, car elle ne nous présente la réalité que comme une série de tableaux morts, étalée, divisée, mesurée dans l'espace.

C'est encore, selon le psychologue, une mémoire froide et sans couleurs, uniforme et conventionnelle, car, si elle permet de feuilleter à discrétion le passé, comme un livre d'images, si même elle affirme *la différence des sensations", en réalité elle ne fait pourtant *que combiner entre eux des éléments bomogènes " ${ }^{12}$, des abstractions. Proust va même jusqu'à la comparer "aux mauvais peintres [qui donnent à nos souvenirs], à tout notre passé étendu sur une

9. BeRgSon, L'Énergie spirituelle, Paris, F. Alcan, 1920, pp. 101-102.

10. Mon ceuvre, dira-t-il, est dominée par la distinction entre la mémoire invo. lontaire et la mémoire volontaire, distinction qui non seulement ne figure pas dans l'auvre de M. Bergson, mais est même contredite par elle. * (Robert DREYFUS, Souvenirs sur Marcel Proust, Paris, B. Grasset, 1936, p. 289.)

11. Ibid., p. 290

12. III (TR), 873 
même toile, les tons conventionnels et tous pareils"13. Mémoire exclusivement pratique, poursuit Proust, lorsqu'elle revient sur le passé pour en utiliser quelques fragments, c'est en leur retirant encore de leur réalité, puisque nous ne conservons alors d'eux que "ce qui convient à la fin utilitaire, étroitement bumaine " ${ }^{14}$ que nous leur assignons. En effet,

... de nos souvenirs relatifs à une personne, l'intelligence élimine tout ce qui ne concourt pas à l'utilité immédiate de nos relations quotidiennes [...]. Elle laisse filer la chaîne des jours passés, n'en gardant fortement que le dernier bout souvent d'un tout autre métal que le dernier chaînon disparu dans la nuit, et dans le voyage que nous faisons à travers la vie, ne tient pour réel que le pays où nous sommes présentement. 15

Aussi Proust est-il persuadé que de tels modes d'appel au passé finissent par l'abolir, au lieu de le conserver :

Au grand jour de la mémoire habituelle [dit-il] les images du passé pâlissent peu à peu, s'effacent, il ne reste plus rien d'elles, nous ne les retrouverons plus. ${ }^{16}$

Les renseignements que cette mémoire nous donne sur le passé "ne conservent rien de lui", puisque de ce passé elle ne nous laisse qu'une image desséchée, une forme vidée de son contenu. Incapable de recréer une tranche véritable de notre vie, elle ne peut fournir des événements qui la jalonnent qu'une évocation sèche, pauvre et mesquine.

On peut déjà s'y attendre: cette forme de mémoire, fonction de l'évocation abstraite, objective et "quasi-dépersonnalisée ", n'a pas, selon Proust, l'aptitude de faire revivre ce passé véritable qu'il recherche dans la plénitude totale. Comme il dit :

Entre l'impression vraie que l'on a eue d'une chose et l'impression factice que l'esprit en donne quand volontairement on essaie de se la représenter, la différence est extrême. ${ }^{17}$

De plus, cette mémoire ne nous représente pas le passé dans sa plénitude totale, mais seulement dans son incomplétude fragmentaire; dès lors, elle ne peut pas nous conduire à la personnalité profonde dont elle ne nous

13. II (G), II.

14. III (TR), 872

15. I (JF), 949.

16. Ibid., p. 643

17. III (TR), 869 
donnerait qu'une image artificielle et factice, privée de son sens véritable et réel.

D'après Proust, la mémoire véritable est la mémoire involontaire, car c'est précisément "lout un instant de la vie [...] pur et désincarné » ${ }^{18}$ qu'elle ressuscite en nous. Bien que ses manifestations soient plus rares, elle seule met vraiment le passé à notre portée en nous le faisant revivre. Sans doute, la mémoire volontaire est bien la mémoire utile, celle qui tient le plus de place dans notre vie au jour le jour, celle qui, dans la plupart des cas, règle l'usage que nous faisons de notre passé. Mais, pour notre chercheur, la mémoire involontaire, bien qu'elle puisse être considérée comme une mémoire gratuite, une mémoire de luxe, est pourtant la mémoire authentique. À l'encontre de la mémoire volontaire, qui ne porte pas sur l'intégrité d'une situation donnée, mais seulement sur tel ou tel point précis, elle nous fait revivre d'une manière souvent inattendue un moment du passé dans sa vérité originale et totale. Avec elle, le passé conserve "toute son épaisseur", "sa densité charnelle et vivante" qui contraste si fortement avec les pauvres schémas stylisés de la mémoire pragmatique. Mémoire de toute la personne, grâce à elle, nous pouvons devenir, en un instant, ce que nous étions auparavant par l'évocation du passé :

Les jours anciens recouvrent peu à peu ceux qui les ont précédés, ils sont eux-mêmes ensevelis sous ceux qui les suivent. Mais chaque jour ancien est resté déposé en nous comme dans une bibliothèque immense où il $\mathrm{y} a$, des plus vieux livres, un exemplaire que sans doute personne n'ira jamais demander. Pourtant que ce jour ancien, traversant la translucidité des époques suivantes, remonte à la surface et s'étende en nous qu'il couvre tout entier, alors pendant un moment, les noms reprennent leur ancienne signification, les êtres leur ancien visage, nous notre âme d'alors, et nous sentons, avec une souffrance vague mais devenue supportable et qui ne durera pas, les problèmes devenus depuis longtemps insolubles et qui nous angoissaient tant alors. ${ }^{19}$

Ainsi donc, d'après le romancier, la connaissance de notre personnalité véritable ne peut nous être fournie que par la mémoire, car, explique-t-il,

... notre moi est fait de la superposition de nos états successifs Mais cette superposition n'est pas immuable comme la strati- 
fication d'une montagne. Perpétuellement des soulèvements font affleurer à la surface des couches anciennes. ${ }^{20}$

Toutefois, précise-t-il, ce n'est pas «la mémoire des faits qui nous dit "tu étais tel», sans nous permettre de le redevenir" ${ }^{21}$, qui nous permettra d'accéder à cette connaissance. Seule la mémoire involontaire, celle qui n'est pas mécanisée par la raison, par l'exercice conscient de l'intelligence, peut faire ce prodige et nous restituer notre moi réel, car elle s'exerce après que l'oubli a mis le souvenir dans un refuge inviolé où il se conserve avec «sa netteté et sa fraîcheur». Et,

... c'est grâce [à elle] que nous pouvons de temps à autre retrouver l'être que nous fûmes, nous placer vis-à-vis des choses comme cet être l'était, souffrir à nouveau, parce que nous ne sommes plus nous, mais lui $[\ldots],{ }^{22}$

Cependant, si Marcel Proust semble concevoir la mémoire involontaire comme le seul moyen d'atteindre le moi profond, il lui reste encore un point important à éclaircir.

Alors que l'évocation usuelle de nos souvenirs habituels répond à une exigence précise du présent, se met à notre disposition à un commandement de la volonté et de l'intelligence, il en va tout autrement des souvenirs involontaires.

Car aux troubles de la mémoire sont liées les intermittences du cour. C'est sans doute l'existence de notre corps, semblable pour nous à un vase où notre spiritualité serait enclose, qui nous induit à supposer que tous nos biens intérieurs, nos joies passées, toutes nos douleurs sont perpétuellement en notre possession. Peut-être est-il aussi inexact de croire qu'elles s'échappent ou reviennent. En tous cas si elles restent en nous, c'est la plupart du temps dans un domaine inconnu où elles ne sont de nul service pour nous, et où même les plus usuelles sont refoulées par des souvenirs d'ordre différent et qui excluent toute simultanéité avec elles dans la conscience. ${ }^{23}$

Sans doute, les souvenirs involontaires remontent au champ de la conscience, s'offrent à notre contemplation,

20. Ibid., pp. 544-45.

21. PM, 97.

22. I (JF), 643 .

23. II (SG), 756-57 
mais c'est toujours à l'improviste, sans avoir été rappelés consciemment. Et même, dit Proust,

c'est peine perdue que nous cherchions à [les] évoquer. Tous les efforts de notre intelligence sont inutiles. Ils sont cachés hors de son domaine et de sa portée. ${ }^{24}$

Comment donc appréhender ces souvenirs qui portent la partie la plus profonde et la plus essentielle de notre personnalité ?

Au dire de Proust, nos souvenirs abandonnés et restés hors de la conscience se désagrègent et se perdent dans l'oubli. Mais, si leurs formes sont abolies, ou du moins, ensommeillées, si elles perdent *leur force d'expansion qui leur eût permis de rejoindre la conscience ${ }^{25}$, il y a quelque chose qui subsiste et leur survit : ce sont les sensations.

Quand d'un passé ancien, [dit-il] rien ne subsiste, après la mort des êtres, après la destruction des choses, seules, plus frêles mais plus vivaces, plus immatérielles, plus persistantes, plus fidèles, l'odeur et la saveur restent encore longtemps, comme des âmes, à se rappeler, à attendre, à espérer, sur la ruine de tout le reste, à porter sans fléchir, sur leur gouttelette presque impalpable, l'édifice immense du souvenir. ${ }^{26}$

Ainsi, selon Proust, le passé continue de vivre dans «une saveur", dans «une odeur»; il est caché "en quelque objet matériel (en la sensation que nous donnerait cet objet matériel) que nous ne soupçonnons pasm. $27 \mathrm{Et}$, si nous pouvons quelque jour, par hasard, donner à nos souvenirs involontaires le support d'une sensation présente, alors ils reprennent vie.

Le point de départ du souvenir involontaire est donc une sensation oubliée qui se réveille, fraîche et active, ce qui soulève de proche en proche, jusqu'au fond de notre inconscient, les souvenirs de notre vie passée. Comme le dit Proust: "Les éléments de notre passé constituent un domaine inconnu qui ne nous est restituable que par la résurrection des impressions qui leur ont servi de cadre " 28

Déjà, dans Jean Santeuil, le psychologue avait soupçonné le pouvoir évocateur des sensations présentes sur les souvenirs passés :

24. I (S), 44

25. Ibid., p. 47

26. Loc. cit.

27. Ibid., p. 44

28. II (SG), 756-57 
De ces choses [écrit-il] qui nous charmèrent autrefois et qu'il nous est donné de revoir, est-il bien vrai qu'il n'y en ait pas certaines, dont la présence nous rende avec une volupté pareille et peut-être plus rêveuse encore le charme mystérieux d'autrefois ? Qu'est-ce donc que ces petites ombres noires flottant à terre sur le chemin rayonnant de soleil, [...] ces vastes arbres fruitiers, $[\ldots]$ qu'est-ce donc que tout cela sinon des témoins de nos premiers printemps, des reliques des souvenirs de nos premières émotions en face de la nature, mais qui n'ont rien perdu de leur pouvoir sur nous, qui ouvrent soudain notre cœur aux mêmes félicités délicieuses qui nous font échapper aux années pour nous rendre à la nature, aux transformations mystérieuses de l'année qui baignent les choses et les événements autour de nous dans une sorte de vie plus grande qu'eux, que nous reconnaissons pour en avoir approché déjà autrefois, qui n'est pas dans notre jeunesse plutôt que dans notre vieillesse et qui pour un moment semble nous montrer le monde médiocre, bientôt fini pour nous, tout humain et connu, mais comme un monde éternel, éternellement jeune, mystérieux, plein de promesses inouïes ? 29

Comme le lui ont appris ces impressions bienheureuses, un aspect de l'expérience actuelle a le pouvoir d'évoquer une expérience antérieure qui, intervenant alors dans la conscience, l'envahit brusquement. Et alors, une sorte de *consonance* entre le passé et le présent s'établit; le "présent d'autrefois", que nous pouvions croire aboli, surgit et s'insère dans le "présent d'aujourd'hui ", jusqu'à le dépouiller presque de son actualité.

Dans $\dot{A}$ la rechercbe du temps perdu, des expériences semblables sont multiples et chaque fois que Proust nous parle d'un souvenir involontaire, nous retrouvons toujours, à l'origine de son apparition, une sensation. Ainsi dans l'expérience de la "madeleine " qui doit, pour la première fois, lui révéler l'existence du souvenir involontaire, c'est le goût du thé qui devait faire surgir le souvenir :

Je portai à mes lèvres [raconte le Narrateur] une cuillerée du thé où j'avais laissé s'amollir un morceau de madeleine. Mais à l'instant même où la gorgée mêlée des miettes du gâteau toucha mon palais, je tressaillis, attentif à ce qui se passait d'extraordinaire en moi. ${ }^{30}$

Et Proust, voulant remettre à sa disposition ce souvenir qui lui avait apporté tant de joie et de bonheur, pour «un éclaircissement décisif $»$, comme il dit, fait encore appel à

29. JS (3), 158-159.

30. I (S), 45 . 
ce breuvage et à sa saveur. Et c'est en ces termes qu'il décrit cette seconde résurgence du souvenir :

Puis une deuxième fois, je fais le vide devant [mon esprit] je remets en face de lui la saveur encore récente de cette première gorgée et je sens tressaillir en moi quelque chose qui se déplace. ${ }^{31}$

Cette résurgence inattendue du souvenir involontaire dans la conscience devait se renouveler à plusieurs reprises et à diverses époques de la vie de Proust. Mais, si le souvenir engendre toujours en lui un sentiment de félicité, de paix et d'éternité, sur lequel il insiste tant, deux choses différeront pourtant: ce sont les sensations à la base de cette évocation et les images évoquées par ces souvenirs. Les sensations sont extrêmement variées et les plus longuement décrites dans son œuvre sont : la vue des clochers de Martinville, la saveur de la madeleine trempée dans une infusion de thé, le tintement d'une cuiller, le fait de buter contre des pavés mal équarris, les deux dalles inégales du baptistère de Saint Marc, la raideur de la serviette à la matinée Guermantes. Toutes ces sensations ont, cependant, le même effet : ressusciter des souvenirs involontaires. «La différence purement matérielle, nous dit Proust, était dans les images évoquées $\# .32$

Pourtant, il faut comprendre ici que l'essentiel de ce phénomène singulier, selon Proust, ne consiste pas dans le mécanisme extérieur, autrement dit, dans la sensation. Malgré cette étroite liaison qu'il semble placer entre la sensation et le souvenir involontaire, il n'y a pas de doute pour lui que le souvenir dépasse infiniment la sensation et surtout n'est pas de même nature qu'elle. Parlant de l'expérience de la madeleine, il dira explicitement : "Je sentais que le /souvenir involontaire Jétait lié au goût du thé et du gâteau, mais qu'il] le dépassait infiniment, ne devait pas être de même nature .33

Ainsi, au dire même de l'écrivain, le gâteau trempé dans du thé, n'est à aucun degré la cause réelle de l'expérience qui lui fut offerte. À peine en est-elle une condition. En fait, bien souvent, tout au long de sa vie, Proust a dû goûter la même chose. Une fois seulement, cette sensation extérieure a exercé sa magie. Il fallait, pour que ce petit signe offert par le présent, cette invitation au passé, fût entendu, que Proust 
se trouvât dans un état de réceptivité tout à fait particulière. En somme, c'est la situation d'ensemble qui commande dans ces cas. La preuve nous en est donnée dans Le temps retrouvé . Le Narrateur y raconte comment dans la cour de l'hôtel de Guermantes, après avoir lutté contre des pavés mal équarris, un souvenir involontaire l'avait envahi pour un instant, mais avait disparu.

... Sans oser plus bouger $[\ldots]$ je restais, quitte à faire rire la foule innombrable des wattmen, à tituber comme j'avais fait tout à l'heure, un pied sur le pavé plus élevé, l'autre pied sur le pavé plus bas. Chaque fois que je refaisais rien que matériellement ce même pas, il me restait inutile mais si je réussissais, oubliant la matinée Guermantes, à retrouver ce que $j$ 'avais senti en posant ainsi mes pieds, de nouveau la vision éblouissante et indistincte me frôlait comme si elle m'avait dit : "Saisis-moi au passage si tu en as la force, et tâche à résoudre l'énigme de bonheur que je te propose. ${ }^{34}$

Comme ce passage le prouve bien, la sensation ou l'impression, qui peut être très pittoresque en soi, n'a, en réalité, qu'une valeur très restreinte dans le souvenir involontaire. Elle est utile, sans doute, puisque sa présence semble indispensable pour son évocation, mais elle n'a rien d'une cause réelle et demeure surtout hors de proportion avec l'expérience qu'elle introduit.

Voilà pourquoi, selon Proust, l'impression "causale» peut être une sensation quelconque. Et même, semble-t-il croire, les sensations objectivement et pratiquement les moins importantes, que nous pensions oubliées, représentent la meilleure sauvegarde du passé35.

... Ce qui nous rappelle le mieux un être [écrit-il] c'est justement ce que nous avions oublié (parce que c'était insignifiant et que nous lui avons ainsi laissé toute sa force). C'est pourquoi la meilleure part de notre mémoire est hors de nous, dans un souffle pluvieux, dans l'odeur de renfermé d'une chambre ou dans l'odeur d'une première flambée, partout où nous retrouvons de nous-même ce que notre intelligence, n'en ayant pas l'emploi, avait dédaigné, la dernière réserve du passé, la meilleure, celle qui, quand toutes nos larmes semblent taries, sait nous faire pleurer encore. Hors de nous ? En nous pour mieux dire, mais dérobée à nos propres regards, dans un oubli plus ou moins prolongé. ${ }^{36}$

34. III (TR), 867

35. Si dans son œuvre, pourtant, le romancier semble faire intervenir le plus souvent les saveurs et les odeurs a l'origine de l'apparition de ces souvenirs, c'est qu'il a été un asthmatique, sensible à un degré inconcevable aux odeurs et aux saveurs les plus minimes. Cela n'implique pas que ces sensations bénéficient, d'après lui, d'un privilège exclusif.

36. I (JF), 643. 
La sensation n'est, par conséquent, que le détail qui déclenche le retour du souvenir involontaire. Malgré les apparences, le souvenir ne nous atteint pas du dehors, mais bien du dedans, "en nous". Peu importe le véhicule du souvenir : il s'efface aussitôt pour faire place à la réalité qui s'est incarnée à travers lui.

\section{III}

DE CE QUI PRÉCÈDE, il ressort déjà que la mémoire involontaire, dans l'œuvre de Proust, n'est pas tout simplement un phénomène dû au hasard, et sur lequel il aurait insisté uniquement par fantaisie ou par souci d'originalité. L'évocation des souvenirs involontaires, qui jalonnent d'un bout à l'autre À la recberche du temps perdu, et qui constituent le thème fondamental dans Le temps retrouvé, dépend, sans doute, comme nous le faisions remarquer plus haut, de rencontres fortuites et même insignifiantes capables de déclencher des apparitions inopinées d'un passé lointain. Mais cette évocation comporte aussi un sens tout à fait précis dans l'œuvre de Proust et joue un rôle unique dans sa vie.

Qu'on ne s'y trompe pas. Cette évocation se distingue absolument d'une logique extérieure où les souvenirs s'appelleraient les uns les autres, selon des lois d'association plus ou moins fixes. Elle n'a pas non plus un caractère passif et contemplatif. Selon le psychologue, dans les souvenirs qui nous reviennent, nous retrouvons un sens qui jusque-là ne nous était pas apparu, le sens le plus profond de la mémoire dans ce regroupement de toute la personnalité, à la faveur des expériences qui furent siennes. On trouve, de ce fait, dans $\grave{A}$ la recherche $d u$ temps perdu, un exemple capable d'étayer définitivement le contenu réel du souvenir involontaire. Le Narrateur vient d'arriver à Balbec pour un nouveau séjour :

Bouleversement de toute ma personne [écrit-il]. Dès la première nuit, comme je souffrais d'une crise de fatigue cardiaque, tâchant de dompter ma souffrance, je me baissai avec lenteur et prudence pour me déchausser. Mais à peine eus-je touché le premier bouton de ma bottine, ma poitrine s'enfla, remplie d'une présence inconnue, divine, des sanglots me secouèrent, des larmes ruisselèrent de mes yeux $[\ldots]$ Je venais d'apercevoir, dans ma mémoire, penché sur ma fatigue, le visage tendre, préoccupé et déçu de ma grand-mère, $[\ldots]$ non pas de celle que je m'étais étonné et reproché de si peu regretter et qui n'avait d'elle que le nom, mais de ma 
grand-mère véritable dont, pour la première fois depuis les Champs-Élysées où elle avait eu son attaque, je retrouvais dans un souvenir involontaire et complet la réalité vivante. ${ }^{37}$

Ainsi, le Narrateur retrouve un jour, tout à fait à l'improviste, la mort de sa grand-mère et la découvre dans la plénitude de sa signification réelle et vivante pour lui. Jusque-là, cette mort ne l'avait pas touché. Sans doute, il savait que sa grandmère était morte, mais son inconscient refusait de le croire. L'événement ne l'avait pas atteint réellement, il était demeuré extérieur comme une donnée sociale et abstraite, dont le sens véritable lui avait échappé. Il faut le retour à Balbec, le retour d'une certaine atmosphère, pour que, d'un coup, la réalité de cette disparition s'inscrive enfin dans son existence.

Mais ce n'est pas tout. Cette restitution d'un passé vécu a encore un sens plus profond pour lui. En effet,

l'être qui venait à mon secours, [continue-t-il] qui me sauvait de la sécheresse de l'âme, c'était celui qui, plusieurs années auparavant, dans un moment de détresse et de solitude identiques, dans un moment où je n'avais plus rien de moi, était entré, et qui m'avait rendu à moi-même, car il était moi et plus que moi. ${ }^{38}$

On le voit, le souvenir involontaire ne représente pas une réalité impersonnelle que l'individu aurait à subir. L'homme ne subit pas passivement l'influence de sensations qui lui renverraient ainsi, de temps en temps, des aspects étrangers à sa vie antérieure. Chaque souvenir représente un instant de la réalité, plus exactement un moment de notre vie personnelle, dont chacun, dans sa constitution même, porte la marque de notre personnalité profonde. Le passé contient le fond même de notre personnalité, il exprime ce que nous sommes, et revient pour nous restituer à nous-mêmes, pour nous permettre de mieux nous comprendre et de ressaisir, de l'avis de Proust, notre «moi ancien ", notre "plus que moi».

La mémoire, chez lui, n'apparaît donc pas sous la forme d'un domaine inerte où le souvenir s'éterniserait dans une immobilité sans substance. Le souvenir involontaire, c'est notre passé.

«La mémoire, affirme Proust, en réveillant les sensations du passé, nous restitue un moi qui n'existait plus qu'à l'état de virtualités *39. 
L'histoire de chaque personne est basée sur des souvenirs qui possèdent pour elle une sorte de valeur constituante. Les événements, que ces souvenirs remémorent, ne se sont pas passés seulement en dehors d'elle. Ils tirent leur signification de ce qu'ils sont aussi des manifestations de tel ou tel aspect de son individualité profonde et lucide. Dans ces souvenirs, le moi véritable se découvre, se retrouve; ils offrent à la personne une image à laquelle elle peut se reporter pour la juger elle-même.

Et, dans le cas de Proust, le souvenir, comme il l'a bien vu, c'est lui-même dans son passé, le moi authentique et sa durable valeur. L'histoire de ses souvenirs n'est autre chose que l'histoire de sa personnalité qu'il essaie de démêler, à travers ce qui n'est pas véritablement lui.

Car, le souvenir involontaire, par la reviviscence totale d'un instant du passé, nous met réellement en présence de l'être que nous fûmes. Par lui, nous retrouvons dans sa réalisation absolue, nos joies, nos souffrances d'alors; nous revoyons intégralement notre personnalité d'autrefois que nous croyions perdue pour toujours.

Bien plus [ajoute Proust] une chose que nous vîmes à une certaine époque, un livre que nous lûmes ne restent pas unis à jamais seulement à ce qu'il y avait autour de nous ; il le reste aussi fidèlement à ce que nous étions alors, il ne peut plus être repassé que par la sensibilité, par la personne que nous étions alors. ${ }^{40}$

Le jeu de la mémoire involontaire, chez Proust, a donc un sens précis et nous renvoie "à nous-même, plutôt qu'à l'événement passé». La mémoire involontaire n'existe pas en dehors de nous; elle obéit aux grands rythmes de notre existence. Malgré les apparences, nous ne nous souvenons pas des choses ou des événements, mais toujours de nousmêmes, sous le voile des choses ou des événements. Henri Delacroix donne du souvenir une définition qui correspond très exactement à celle de Proust: «Un souvenir est un fragment de vie personnelle dont l'évocation tend à la réactivation du moi passé. Mais le moi passé commence par se refaire présent avant de s'évoquer comme passé. Il dé. borde [...] le présent qui l'évoque». ${ }^{41}$

Le vrai rôle du souvenir consiste donc à démasquer la personnalité profonde, car le souvenir, en nous faisant

40. III (TR), 885 .

41. DELACROIX, Henri, Les grandes formes de la vie mentale, Paris, F. Alcan, 1934, p. 120 . 
revivre un moment du passé, nous apporte le signe d'une réalité qui dépasse l'empirique. Un moment du passé vient de revivre. «Rien qu'un moment du passé ? se demande Proust. Beaucoup plus, peut-être; quelque chose qui, commun à la fois au passé et au présent, est beaucoup plus essentiel qu'eux deux ${ }^{42}$. Mais quel est-il *ce quelque chose de beaucoup plus essentiel " que le passé et le présent qui charge de toute son intensité, de toute sa présence le moment où il a pu intervenir ?

Tant de fois, au cours de ma vie, la réalité m'avait déçu parce qu'au moment où je la percevais, mon imagination, qui était mon seul organe pour jouir de la beauté, ne pouvait s'appliquer à elle, en vertu de la loi inévitable qui veut qu'on ne puisse imaginer que ce qui est absent. Et voici que soudain l'effet de cette dure loi s'était trouvé neutralisé, suspendu, par un expédient merveilleux de la nature, qui avait fait miroiter une sensation - bruit de la fourchette et du marteau, même titre de livre, etc. - à la fois dans le passé, ce qui permettait à mon imagination de la goûter, et dans le présent où l'ébranlement effectif de mes sens par le bruit, le contact du linge, etc. avait ajouté aux rêves de l'imagination ce dont ils sont habituellement dépourvus, l'idée d'existence, et, grâce à ce subterfuge, avait permis à mon être d'obtenir, d'isoler, d'immobiliser - la durée d'un éclair - ce qu'il n'appréhende jamais : un peu de temps à l'état pur. ${ }^{43}$

Évidemment, le "temps à l'état pur", dont il est ici question, c'est bien, plutôt, la personnalité à l'état pur, déliée de ses engagements, par l'expérience même de leur répétition ; c'est le passage, en quelque sorte, du temps de la personne à son éternité, à son affirmation intemporelle. Proust insiste sur ce point :

Qu'un bruit, qu'une odeur, déjà entendu ou respirée jadis, le soient de nouveau, à la fois dans le présent et dans le passé, réels sans être actuels, idéaux sans être abstraits, aussitôt l'essence permanente et habituellement cachée des choses se trouve libérée, et notre vrai moi qui, parfois depuis long. temps, semblait mort, mais ne l'était pas entièrement, s'éveille, s'anime, en recevant la céleste nourriture qui lui est apportée. Une minute affranchie de l'ordre du temps a recréé en nous, pour la sentir, l'homme affranchi de l'ordre du temps. ${ }^{44}$

Ainsi donc, dans l'œuvre de Proust, le souvenir involontaire est considéré moins comme l'évocation tout simple-

42. III (TR), 872 .

43. Loc. cit.

44. III (TR), 872-73. 
ment d'un fait passé que comme la restitution à "l'bomme affranchi de l'ordre du temps", de son "vrai moi [...] qui semblait mort, mais ne l'était pas. "Le Narrateur, dans A la recherche du temps perdu, retrouvant le visage ancien de sa grand-mère penchée sur lui, dans un jour semblable de détresse, précise bien que le retour du passé dans le souve. nir involontaire est un retour du moi :

Le moi que j'étais alors, et qui avait disparu si longtemps, était de nouveau si près de moi qu'il me semblait encore entendre les paroles qui avaient immédiatement précédé et qui n'étaient pourtant plus qu'un songe. [...] Je n'étais plus que cet être qui cherchait à se réfugier dans les bras de sa grand'mère, à effacer les traces de ses peines en lui donnant des baisers. ${ }^{45}$

Le souvenir involontaire chez Proust se caractérise par l'affirmation du primat de la personnalité et de ses valeurs sur les éléments représentatifs. Il conserve non pas un canevas desséché, mais la plénitude même du moi profond. Et cette possibilité de le ressaisir, par l'intermédiaire du souvenir involontaire, existe constamment en nous; elle n'attend que l'occasion propice, au dire de l'écrivain, pour émerger à la surface de la monotonie de notre présent. ${ }^{46}$

Mais, ici encore, il faut comprendre que dans cette affirmation du primat de la personnalité, il ne s'agit pas d'une simple fantaisie de la part de Proust, encore moins d'une hallucination.

Le «moi ancien» et le «moi présent» s'appellent l'un l'autre, en vertu d'une «consonance essentielle». Une nécessité les relie qui doit nous permettre de pénétrer plus avant dans les rapports intimes qui existent entre la mémoire involontaire et la personnalité profonde.

Malgré les apparences, en effet, le souvenir involontaire ne se suffit pas à lui-même. Il n'est jamais la résurgence pure et simple du passé. Chez Proust, il prend un sens particulier et profond : il indique une coïncidence de son «moi ancien * et de son "moi présent" qui, du même coup, l'élève au niveau d'une sorte de transcendance personnelle. Sa personnalité s'affirme en se redoublant, en se confirmant ellemême sous le détour des circonstances. Lui-même a bien vu

45. II (SG), 757.

46. Toutefois, précise-t-il, nous ne sommes pas toujours disposés à recevoir la visite des souvenirs involontaires et du moi qu'ils nous révèlent, car * [...] chaque fois, [note le Narrateur] la lacheté qui nous détourne de toute tacbe difficile, de toute auvre importante, m'a conseille de laisser cela, de boire mon the en pensant simplement a mes ennuis d'aujourd'bui, à mes désirs de demain qui se laissent remacber sans peine *. (I (S), 46). 
ce qu'il y avait d'extraordinaire dans ses expériences, et voici ce qu'il écrit, à propos du goût de la petite madeleine :

Au vrai, l'être qui alors goûtait en moi cette impression la goûtait en ce qu'elle avait de commun dans un jour ancien et maintenant, dans ce qu'elle avait d'extra-temporel, un être qui n'apparaissait que quand, par une de ces identités entre le présent et le passé, il pouvait se trouver dans le seul milieu où il pût vivre, jouir de l'essence des choses, c'est-à-dire en dehors du temps. ${ }^{47}$

Mais, pour que deux impressions semblables, séparées par une longue période de temps, puissent se superposer dans une même conscience, il faut que cette conscience présente elle-même un fond permanent. Et il reste que «l'extra-temporel», «l'essence» dont il s'agit n'appartiennent pas à l'objet, ainsi qu'on pourrait le croire, mais uniquement au sujet. C'est son moi, le plus profond, son propre affranchissement de la loi du temps qu'entrevoit Proust dans cet instant. Et dans ce phénomène de mémoire, les détails minutieux que le souvenir involontaire a sauvegardés ne valent pas en eux-mêmes. Les faits passés ne sont pas ressentis comme ayant fait partie simplement de son moi antérieur, mais bien comme une présence réelle attachée à son moi absolu et permanent. Proust y voit l'intuition de lui-même vivant sans être soumis aux lois du temps; il se perçoit comme identique à ce qu'il était autrefois.

Sans doute notre personnalité profonde ne se donne-telle pas à nous immédiatement dans l'évocation des souvenirs, puisqu'elle demeure inaccessible à tout effort direct pour la ressaisir. Mais elle paraît plus accessible à la faveur d'expériences privilégiées qu'il nous est donné de vivre.

Dans les souvenirs évoqués, c'est lui-même, son moi véritable, que Proust affirme, visant à travers les détails remémorés, une plus complète réalisation de sa personne. De là le caractère en quelque sorte contradictoire de la description qui nous en est faite. L'auteur, en effet, parle d'un foisonnement indéfini de détails authentiques, s'appelant les uns les autres, et qui doivent occuper dès lors une certaine durée. Pourtant, les descriptions de cette expérience insistent toutes sur son caractère extrêmement bref qui contraste tout à fait avec l'idée d'un prolongement dans le temps.

C'est ainsi que dans le passage déjà cité, où il raconte l'incident de la madeleine, Proust évoque «ce souvenir, l'instant ancien que l'attraction d'un instant identique est

47. III (TR), 871 
venue de si loin solliciter, émouvoir, soulever tout au fond de moi ${ }^{48}$ De même, dans d'autres passages analogues, les mots «soudain», "aussitôt», reviennent sans cesse pour rendre compte de l'événement. La reviviscence du passé se réalise dans un instant. C'est pourquoi, d'ailleurs, Proust peut parler à son propos, "d'une minute affranchie de l'ordre du temps».49 Il s'agit bien d'une expérience intemporelle qui s'oppose à la durée : c'est l'instant du souvenir involontaire. Forme extrême du temps, discontinuité, saut par la vertu duquel, rompant avec un certain ordre d'existence, nous nous installons dans un autre, d'une valeur supérieure, "l'instant proustien " apparaît comme le véhicule de toute révélation de la personnalité profonde. Cet instant vient de cette conscience d'identité qu'on ressent à ce moment, de l'intuition par laquelle on se perçoit à la fois comme passé et comme présent, par laquelle on revit sans avoir l'impression de n'être plus ce qu'on était autrefois. À ce point, on le sent bien, le souvenir involontaire, qui engendre chez Proust ce sentiment de libération du temps, de la contingence et du néant, prend un sens non plus chronologique et temporel, mais métaphysique et " extratemporel ", pour lui emprunter cette dernière expression.

Une dernière preuve nous est fournie, dans cette impression de bonheur, de satisfaction et de félicité intenses qui semble toujours accompagner en lui l'évocation du souvenir involontaire.

À première vue, cette sensation de plénitude et d'euphorie ne semble pas correspondre en fait à un aspect du phénomène passé. Trois arbres dans la campagne, la saveur d'une madeleine ou la disparité de deux pavés inégaux sous le pied renvoient sans doute à des impressions antérieures, mais elles ne possèdent sûrement pas cette vertu, cette puissance, dont elles se révèlent subitement douées. Ce n'est pas non plus que l'instant du passé ainsi rappelé ait été lui-même, au moment où il a été effectivement vécu, source de félicité et de joie. Quelle peut donc bien être la cause de cette félicité ?

Cette cause [nous dit Proust] je la devinais en comparant ces diverses impressions bienheureuses et qui avaient entre elles ceci de commun que je les éprouvais à la fois dans le moment actuel et dans un moment éloigné, jusqu'à faire empiéter le passé sur le présent, à me faire hésiter à savoir dans lequel des deux je me trouvais $[\ldots]$ Cela expliquait que mes inquiétudes 
au sujet de ma mort eussent cessé au moment où j'avais reconnu inconsciemment le goût de la petite madeleine, puisqu'à ce moment-là l'être que j'avais été était un être extratemporel, par conséquent insoucieux des vicissitudes de l'avenir. Cet être-là n'était jamais venu à moi, ne s'était jamais manifesté, qu'en dehors de l'action, de la jouissance immédiate, chaque fois que le miracle d'une analogie m'avait fait échapper du présent. Seul, il avait le pouvoir de me faire retrouver les jours anciens, le temps perdu, devant quoi les efforts de ma mémoire et de mon intelligence échouaient toujours. ${ }^{50}$

Il découle de là que ce n'est pas tant le souvenir qui comporte en soi cette joie et cette félicité, mais le souvenir en tant que fonction expressive et libératrice de la personnalité profonde. «Chaque souvenir, en effet, met en jeu notre moi, nous énonce et nous donne à nous-mêmes". Étant l'expression du moi, sa plénitude correspond à la mesure dans laquelle il accomplit cette fonction expressive. Proust, nous le savons, se recherchait lui-même, se visait à travers ses souvenirs. Et lorsqu'il s'est ainsi réalisé, il a l'impression de s'être libéré, de s'être affirmé au-dessus du temps, de l'oubli et de la mort. D'où ce sentiment de joie et de satisfaction, qui accompagne presque toujours l'évocation du souvenir involontaire chez lui.

En fait, le souvenir n'a aucune part réelle dans cette joie, mais il répond comme à une sorte de besoin du moi profond, qui réclame satisfaction de reconnaissance. Il apporte à l'auteur du Temps retrouvé, après des années d'inquiétude et de doute de soi, la possibilité de retrouver une richesse perdue : la personnalité considérée comme un bien personnel d'une exceptionnelle valeur. Ce prestige de la personnalité retrouvée affirme en lui la satisfaction du recouvrement d'une valeur essentielle, qui lui appartient en propre, et entraîne dans le souvenir évoqué cette teneur affective sur laquelle il insiste si fort.

Noël Martin-Deslias l'a bien vu : chez Proust, dit-il, ces instants de bonheur "...qu'il éprouve viennent de cette conscience d'identité, de l'intuition par laquelle il se perçoit à la fois comme passé et comme présent, par laquelle il revit sans avoir l'impression de n'être plus ce qu'il était autrefois. „51

51. MARTIN-DESLIAS, L'Idéalisme de Marcel Proust, Paris, Les Éditions Nagel, 1952 , p. 178 
La conséquence de tout cela reste que la mémoire, selon Proust, du moins la mémoire involontaire, est liée à l'actualité de notre vie par la plus mystérieuse des intimités. Elle a même une fonction métaphysique précise en nous et joue un rôle de premier plan dans la révélation de notre personnalité profonde.

Le souvenir involontaire, à son sens, n'est pas quelque chose qu'on a, c'est quelque chose qu'on est. Sa fonction n'est pas seulement de fixer des événements écoulés, mais de nous exprimer nous-mêmes, d'affirmer l'éternité de notre personnalité authentique.

Dans le cas même du romancier, le souvenir, qui est une sorte de remise en jeu du moi passé, lui permet, en l'opposant à son moi présent et en revenant sur lui, d'en prendre une conscience nouvelle. La mémoire est chez lui l'expression de sa personnalité tout au long de son existence. Elle n'est plus la simple histoire de sa vie personnelle, elle se dépasse elle-même ; et le souvenir involontaire, par sa structure "présent-passé", a pour effet de souligner les traits profonds et les grandes constantes de la personnalité véritable. "Monde fabuleux de nos souvenirs, écrit Proust, qui devient celui de la vérité. Heures qui avaient donné son cour à quelque chose de plus profond que la vanité, de plus durable que l'amour $[\ldots]{ }^{52}$. Le souvenir se donne donc à lui comme un moyen, comme un signe de la vérité, pour exprimer, pour incarner cette forme supérieure de conscience de soi qu'est la personnalité profonde.

La conception de Proust s'écarte ici de celle de Bergson. D'après ce dernier, en effet, nous atteignons notre moi premier et profond par une «réflexion métaphysique», c'està-dire par «l'intuition de la durée : connaissance expérimentale singulière, transcendant l'intelligence et la raison, sympathisant avec le réel, mais fugitive. [Cette intuition de la durée] exige un grand effort de la volonté, où "la faculté de voir tordue sur elle-même ne fait qu'un avec l'acte de vouloir." (Bergson, L'évolution créatrice, p. 258). L'intuition nous fait vibrer avec la réalité dans ses plus intimes profondeurs, elle nous transporte au cœur du réel $[\ldots]$ „ 53 . Comme l'a justement dit Charles Blondel: «Le génie de Proust est une longue mémoire. Cette surprenante imagination mémorialisée, il s'en est servi non seulement pour se

52. JS (2), 225

53. Peillaube, E., Caractère et personnalite, Paris, Pierre Téqui, 1935, p. 157. 
rappeler, mais pour découvrir ${ }^{54}$. Le premier, surtout, il a vu qu'elle était le moyen en possession de l'homme pour communiquer avec son moi profond.

The Graduate School and University Center, The City University of New York

\section{ABRÉVLATIONS}

Pour les références aux nombreuses citations de l'œuvre de Proust, nous utiliserons le système suivant d'abréviations : les chiffres romains renvoient au tome; les lettres majuscules au titre des volumes; dans les références les chiffres qui suivent les observations renvoient aux pages.

I. - Abréviations pour "À la recherche du temps perdu",

N.R.F. Bibliothèque de la Pléiade, 1954, 3 tomes.

Tome I

Du côté de chez Swann I $(S)$

À l'ombre des jeunes filles en fleurs I (JF)

Tome II

Le côté de Guermantes

II $(G)$

Sodome et Gomorrhe

II $(S G)$

Tome III

$\begin{array}{ll}\text { La Prisonnière } & \text { III }(P) \\ \text { La Fugitive } & \text { III }(F) \\ \text { Le temps retrouvé } & \text { III }(T R)\end{array}$

II. - Abréviations pour les autres ouvrages de Proust.

Les plaisirs et les jours

Pastiches et mélanges

PJ

Chroniques

Jean Santeuil, vol. I

Jean Santeuil, vol. 2

Jean Santeuil, vol. 3

Contre Sainte-Beuve

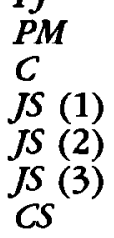

54. BLONDEL, Charles, La psychograpbie de Marcel Proust, Paris, Vrin, 1932, p. 11. 